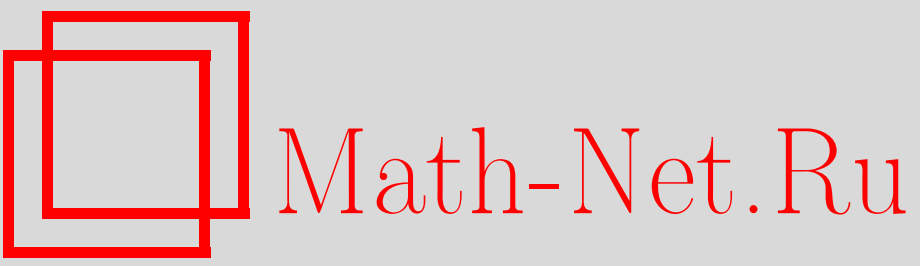

О. Ю. Шведов, О релятивистски-ковариантной квантово-полевой теории комплексного ростка Маслова, ТМФ, 2005, том 144, номер 3, 492-512

DOI: https://doi.org/10.4213/tmf1873

Использование Общероссийского математического портала Math-Net.Ru подразумевает, что вы прочитали и согласны с пользовательским соглашением

http://www . mathnet.ru/rus/agreement

Параметры загрузки:

IP : 54.237 .206 .68

26 апреля 2023 г., 16:03:31 
ТЕОРЕТИЧЕСКАЯ

И МАТЕМАТИЧЕСКАЯ

ФИЗИКА

Том 144, № 3

сентябрь, 2005

(C) 2005 r.

О.Ю. Шведов*

\title{
О РЕЛЯТИВИСТСКИ-КОВАРИАНТНОЙ КВАНТОВО-ПОЛЕВОЙ ТЕОРИИ КОМПЛЕКСНОГО РОСТКА МАСЛОВА
}

\begin{abstract}
Рассматривается явно ковариантная формулировка квантово-полевой теории комплексного ростка Маслова (квазиклассической теории поля) на примере скалярного поля. Основной объект теории - "квазиклассическое расслоение", базой которого является множество классических состояний, слоями - пространства состояний квантовой теории во внешнем поле. Квазиклассические состояния, возникающие в теории комплексного ростка Маслова в точке и в теории лагранжевых многообразий с комплексным ростком, изображаются точками и поверхностями на пространстве квазиклассического расслоения, соответственно. Сформулированы квазиклассические аналоги аксиом квантовой теории поля; установлена взаимосвязь ковариантной квазиклассической теории как с построенной ранее гамильтоновой формулировкой, так и с конструкциями аксиоматической теории поля: источниками Швингера, $S$-матрицей Боголюбова, $R$-функциями Лемана-Симанзика-Циммермана. Предложена новая ковариантная формулировка классической теории поля и схема квазиклассического квантования полей без использования постулата о замене скобок Пуассона на коммутаторы.
\end{abstract}

Ключевые слова: комплексный росток Маслова, аксиоматическая квантовая теория поля, $S$-матрица Боголюбова, подход Лемана-Симанзика-Циммермана, теория источников Швингера, скобки Пайерлса.

\section{1. ВВЕ ДЕНИЕ}

Квазиклассические методы играют важную роль в квантовой механике и теории поля. Наиболее последовательным подходом к квазиклассической механике является разработанная Масловым теория лагранжевых многообразий с комплексным ростком [1], [2]. Она основана на непосредственной подстановке предполагаемой волновой функции в уравнение Шредингера, допускает математически строгое обоснование (оценку погрешности) и включает в себя в качестве частных случаев другие квазиклассические методы, в частности подход Эренфеста, осцилляторное приближение, методы ВКБ и канонического оператора Маслова [3].

При переносе теории комплексного ростка Маслова на квантовую теорию поля следует учитывать специфику последней, связанную как с расходимостями, так и с релятивистской инвариантностью. При этом непосредственное применение методов Маслова

\footnotetext{
* Московский государственный университет, Москва, Россия. E-mail: shvedov@qs.phys.msu.su
} 
возможно только в гамильтоновом подходе к теории поля, в котором на основе классического лагранжиана строится гамильтониан и ему с помощью процедуры канонического квантования сопоставляется уравнение Шредингера. Анализ расходимостей и релятивистской инвариантности в данном подходе затруднен.

Сведения из гамильтоновой квантово-полевой теории комплексного ростка Маслова приведены в разделе 2. Рассматривается скалярное поле $\varphi$ с лагранжианом, зависяшим от малого параметра $h$ ( "постоянной Планка") как

$$
\mathcal{L}=\frac{1}{2} \partial_{\mu} \varphi \partial^{\mu} \varphi-\frac{1}{h} V(\sqrt{h} \varphi)
$$

где потенциал самодействия $V(\Phi) \sim\left(m^{2} / 2\right) \Phi^{2}$ при $\Phi \rightarrow 0$, и соответствующее ему уравнение Шредингера. Метод комплексного ростка Маслова в точке [1], [2] (называемый также методом траекторно-когерентных состояний [4]) заключается в подстановке состояния вида [5], [6]

$$
\Psi(t)=e^{(i / h) \widetilde{S}^{t}} \exp \left\{\frac{i}{\sqrt{h}} \int d \mathbf{x}\left[\Pi^{t}(\mathbf{x}) \hat{\varphi}(\mathbf{x})-\Phi^{t}(\mathbf{x}) \hat{\pi}(\mathbf{x})\right]\right\} f^{t}
$$

при $h \rightarrow 0$ в уравнение Шредингера. Здесь $\widetilde{S}^{t}$ - числовая вешественная функция времени, имеюшая смысл классического действия на временно́м промежутке от 0 до $t, \Pi^{t}(\mathbf{x})$ и $\Phi^{t}(\mathbf{x})$ - классические функции пространственных переменных $\mathbf{x}$ и времени $t, \hat{\varphi}(\mathbf{x})$ и $\hat{\pi}(\mathbf{x})$ - операторы поля и канонически сопряженного импульса, $f^{t}$ - имеющий предел при $h \rightarrow 0$ вектор состояния, зависящий от времени. Функции $\widetilde{S}, \Pi, \Phi, f$ должны удовлетворять определенным соотношениям. В частности, для классических поля $\Phi$ и импульса П получаются классические уравнения движения.

Помимо квазиклассической эволюции, в квазиклассической теории поля строятся преобразования Пуанкаре, операторы поля и другие структуры, необходимые для теории лагранжевых многообразий с комплексным ростком. Их перенормировка в главном порядке разложения рассмотрена в работе [7].

В отличие от гамильтонова подхода, аксиоматическое построение квантовой теории поля [8] в подходах Лемана-Симанзика-Циммермана (ЛСЦ) [9], Боголюбова [10] и Боголюбова-Медведева-Поливанова [11] основано на наиболее общих принципах: релятивистской ковариантности, унитарности и причинности. При этом вся теория строится явно ковариантным образом, что облегчает анализ расходимостей, особенно в высших порядках теории возмущений [10], [12], [13].

В п. 3.1 рассмотрена проблема квазиклассического приближения в аксиоматическом подходе. Вместо состояний (1.2), использующих явное разбиение на пространственные и временны́е компоненты, рассматриваются "сглаженные" по времени векторы состояний вида

$$
\Psi=e^{(i / h) \bar{S}} T \exp \left[\frac{i}{\sqrt{h}} \int d x J(x) \hat{\varphi}_{h}(x)\right] \bar{f} \equiv e^{(i / h) \bar{S}} T_{J}^{h} \bar{f},
$$

где $\bar{S}$ - некоторое число, $\hat{\varphi}_{h}(x)$ - гейзенберговский оператор поля, $J(x)$ - числовая функция, имеюшая смысл внешнего классического источника (используемого в теории источников Швингера [14]), обрашаюшаяся в нуль вне интервала $x^{0} \in\left[T_{-}, T_{+}\right], \bar{f}$ - имеющий предел при $h \rightarrow 0$ вектор состояния. Через Техр обозначена хронологическая экспонента [10]. 
Оказьвается, что в главном порядке по $h$ при $t>T_{+}$справедливо свойство

$$
\hat{\varphi}_{h}(x) T_{J}^{h} \bar{f} \simeq \frac{1}{\sqrt{h}} \bar{\Phi}(x) T_{J}^{h} \bar{f},
$$

причем функция $\bar{\Phi}(x) \equiv \bar{\Phi}_{J}(x)$ является решением задачи Коши

$$
\partial_{\mu} \partial^{\mu} \bar{\Phi}+V^{\prime}(\bar{\Phi})=J,\left.\quad \bar{\Phi}\right|_{t<T_{-}}=0
$$

и имеет смысл классического поля, порождаемого источником $J$.

Как показано в разделе 4 , состояние (1.3) при определенных условиях, связывающих $(\bar{S}, J, \bar{f})$ и $(\widetilde{S}, \Pi, \Phi, f)$, при $h \rightarrow 0$ приближенно совпадает с (1.2). В важном частном случае $T_{+}<0$ функции $\Phi$ и П совпадают соответственно с классическим полем $\bar{\Phi}_{J}$ и его производной по времени при $t=0$.

Специфика ковариантного подхода к квазиклассической теории поля заключается в том, что источники $J_{1} \neq J_{2}$ могут давать одно и то же квазиклассическое состояние (1.3), если порождаемые ими поля $\bar{\Phi}_{J_{1}}$ и $\bar{\Phi}_{J_{2}}$ при $t>T_{+}$совпадают. Учет отношения эквивалентности квазиклассических состояний, напоминаюший учет калибровочной инвариантности в калибровочных теориях [13], рассмотрен в п. 3.2. В частности, если поле $\bar{\Phi}_{J}$ имеет компактньй носитель (обрашается в нуль при $t>T_{+}$), то при $h \rightarrow 0$

$$
T_{J}^{h} \bar{f} \simeq e^{(i / h) \bar{I}} W_{J} \bar{f}
$$

для некоторой фазы $\bar{I}$ и оператора $W_{J}$, имеющего смысл матрицы рассеяния на классическом поле $\bar{\Phi}$.

Положив соотношение (1.6) в основу теории, можно вывести из первых принципов классические уравнения движения (принцип стационарности действия) и коммутационные соотношения для квазиклассических полей без использования постулата о замене скобки Пуассона коммутатором и установить связь аксиоматической теории и гамильтонова формализма. Для оператора $W_{J}$ получаются аналоги свойств релятивистской ковариантности, унитарности, причинности.

\section{2. СВЕДЕНИЯ ИЗ ГАМИЛЬТОНОВОЙ КВАНТОВО-ПОЛЕВОЙ ТЕОРИИ КОМПЛЕКСНОГО РОСТКА МАСЛОВА}

2.1. Рассмотрим уравнение Шредингера для модели с лагранжианом (1.1):

$$
i \frac{d}{d t} \Psi(t)=\int d \mathbf{x}\left[\frac{1}{2} \hat{\pi}^{2}(\mathbf{x})+\frac{1}{2}(\nabla \hat{\varphi}(\mathbf{x}))^{2}+\frac{1}{h} V(\sqrt{h} \hat{\varphi}(\mathbf{x}))\right] \Psi(t),
$$

где $\Psi(t)$ - зависящий от времени вектор состояния (элемент гильбертова пространства $\left.\mathcal{H}^{h}\right), \hat{\varphi}(\mathbf{x})$ и $\hat{\pi}(\mathbf{x})$ - операторы поля и канонически сопряженного импульса, удовлетворяюшие каноническим коммутационным соотношениям. В шредингеровском функциональном представлении состояниям в каждый момент времени сопоставляются функционалы $\Psi[\varphi(\cdot)]$; операторы $\hat{\varphi}$ и $\hat{\pi}$ записываются как $\hat{\varphi}(\mathbf{x}) \equiv \varphi(\mathbf{x}), \hat{\pi}(\mathbf{x}) \equiv-i \delta / \delta \varphi(\mathbf{x})$. Состояние (1.2) представляется как

$$
\begin{aligned}
\Psi[t, \varphi(\cdot)] & =e^{(i / h) S^{t}} \exp \left\{\frac{i}{h} \int d \mathbf{x} \Pi^{t}(\mathbf{x})\left[\varphi(\mathbf{x}) \sqrt{h}-\Phi^{t}(\mathbf{x})\right]\right\} f^{t}\left[\varphi(\cdot)-\frac{\Phi^{t}(\cdot)}{\sqrt{h}}\right] \equiv \\
& \equiv\left(K_{S^{t}, \Pi^{t}, \Phi^{t}}^{h} f^{t}\right)[\varphi(\cdot)],
\end{aligned}
$$


где $S^{t}=\widetilde{S}^{t}+(1 / 2) \int d \mathbf{x} \Pi^{t}(\mathbf{x}) \Phi^{t}(\mathbf{x}), f^{t}-$ не зависящий от $h$ функционал. Подстановка функционала (2.2) в уравнение Шредингера приводит к следующим соотношениям [5], [6]:

а) П, Ф удовлетворяют классическим уравнениям

$$
\dot{\Phi}^{t}=\Pi^{t}, \quad-\dot{\Pi}^{t}=-\Delta \Phi^{t}+V^{\prime}\left(\Phi^{t}\right) ;
$$

б) $S$ - действие на классическом решении, т.е.

$$
\dot{S}^{t}=\int d \mathbf{x}\left[\Pi^{t}(\mathbf{x}) \dot{\Phi}^{t}(\mathbf{x})-\frac{1}{2}\left(\Pi^{t}(\mathbf{x})\right)^{2}-\frac{1}{2}\left(\nabla \Phi^{t}(\mathbf{x})\right)^{2}-V\left(\Phi^{t}(\mathbf{x})\right)\right] ;
$$

в) $f^{t}$ удовлетворяет уравнению Шредингера во внешнем поле $\Phi^{t}(\mathbf{x})$ с квадратичным гамильтонианом

$$
\begin{gathered}
i \dot{f}^{t}[\phi(\cdot)]=H_{2}^{t} f^{t}[\phi(\cdot)] \\
H_{2}^{t}=\int d \mathbf{x}\left[-\frac{1}{2} \frac{\delta^{2}}{\delta \phi(\mathbf{x}) \delta \phi(\mathbf{x})}+\frac{1}{2}(\nabla \phi(\mathbf{x}))^{2}+\frac{1}{2} V^{\prime \prime}\left(\Phi^{t}(\mathbf{x})\right) \phi^{2}(\mathbf{x})\right] .
\end{gathered}
$$

Для устранения квантово-полевых расходимостей в главном порядке квазиклассического разложения нужно выбрать (вообще говоря, нефоковское) представление коммутационных соотношений и добавить к квадратичному гамильтониану, входящему в (2.5), бесконечное числовое слагаемое, зависяшее от классического поля [6], [7].

2.2. Таким образом, состояния, возникающие в квазиклассической гамильтоновой теории комплексного ростка Маслова в точке, можно в каждый момент времени отождествить с упорядоченными парами $(X, f)$, где $X \equiv(S, \Pi, \Phi)$ - классические переменные в фиксированный момент времени, $f$ - квантовое состояние во внешнем поле - элемент некоторого гильбертова пространства $\mathcal{F}_{X}$, зависяшего от $X$. Эволюция определяется как совокупность преобразований $u_{t}: X \mapsto u_{t} X, U_{t}\left(u_{t} X \leftarrow X\right): \mathcal{F}_{X} \rightarrow \mathcal{F}_{u_{t} X}$. Математически множество наборов $(X, f)$ можно проинтепретировать [15] как векторное расслоение с базой $\{X\}$ и слоями $\mathcal{F}_{X}$ ("квазиклассическое расслоение"), а эволюцию - как автоморфизм квазиклассического расслоения. При этом определен оператор $K_{X}^{h}: \mathcal{F}_{X} \rightarrow \mathcal{H}^{h}$, сопоставляющий при каждом $X$ вектору $f$ элемент пространства состояний "точной теории" $\mathcal{H}^{h}$ вида $(2.2)$. Он называется каноническим оператором.

Если $\mathcal{U}_{t}^{h}$ - оператор эволюции в “точной” теории поля, действующий в $\mathcal{H}^{h}$, то можно записать

$$
\mathcal{U}_{t}^{h} K_{X}^{h} f \simeq K_{u_{t} X}^{h} U_{t}\left(u_{t} X \leftarrow X\right) f .
$$

Соотношение (2.6) обобшается и на случай преобразования $\mathcal{U}_{g}^{h}$, соответствуюшего элементу группы Пуанкаре $g \in G$ :

$$
\mathcal{U}_{g}^{h} K_{X}^{h} f \simeq K_{u_{g} X}^{h} U_{g}\left(u_{g} X \leftarrow X\right) f
$$

где

$$
u_{g}: X \mapsto u_{g} X, \quad U_{g}\left(u_{g} X \leftarrow X\right): \mathcal{F}_{X} \rightarrow \mathcal{F}_{u_{g} X}
$$

Построение перенормированных преобразований (2.8) и проверка группового свойства в гамильтоновом подходе проведены в работе [7]. 
Сформулируем аналоги аксиом квантовой теории поля для квазиклассической теории комплексного ростка Маслова в точке в гамильтоновом формализме.

A1. Определено квазиклассическое расслоение, пространство которого является множеством состояний квазиклассической теории, база $\mathcal{X}$ - множеством классических состояний, слои $\mathcal{F}_{X}$ - гильбертовыми пространствами квантовой системы во внешнем поле $X \in \mathcal{X}$.

A2 (релятивистская инвариантность). На квазиклассическом расслоении действует группа Пуанкаре $G$ : әлементам $g \in G$ сопоставлены автоморфизмы (2.8) квазиклассического расслоения, удовлетворяющие групповому свойству:

$$
\begin{aligned}
u_{g_{1} g_{2}} & =u_{g_{1}} u_{g_{2}}, \\
U_{g_{1} g_{2}}\left(u_{g_{1} g_{2}} X \leftarrow X\right) & =U_{g_{1}}\left(u_{g_{1} g_{2}} X \leftarrow u_{g_{2}} X\right) U_{g_{2}}\left(u_{g_{2}} X \leftarrow X\right) .
\end{aligned}
$$

Исследуем аналог аксиомы об операторе поля. Поскольку при $t=0$

$$
\hat{\varphi}(\mathbf{x}) K_{X}^{h} f=K_{X}^{h}\left[\frac{\Phi(\mathbf{x})}{\sqrt{h}}+\hat{\phi}(\mathbf{x})\right] f,
$$

где $\hat{\phi}(\mathbf{x})$ - оператор умножения на $\phi(\mathbf{x})$ в функциональном шредингеровском представлении, для гейзенберговского оператора поля имеем

$$
\begin{aligned}
\hat{\varphi}_{h}(\mathbf{x}, t) K_{X}^{h} f & =\mathcal{U}_{-t}^{h} \hat{\varphi}(\mathbf{x}) \mathcal{U}_{t}^{h} K_{X}^{h} f \simeq \mathcal{U}_{-t}^{h} \hat{\varphi}(\mathbf{x}) K_{u_{t} X}^{h} U_{t}\left(u_{t} X \leftarrow X\right) f= \\
& =\mathcal{U}_{-t}^{h} K_{u_{t} X}^{h}\left[\frac{\Phi^{t}(\mathbf{x})}{\sqrt{h}}+\hat{\phi}(\mathbf{x}) U_{t}\left(u_{t} X \leftarrow X\right)\right] f= \\
& =K_{X}^{h}\left[\frac{\Phi^{t}(\mathbf{x})}{\sqrt{h}}+U_{-t}\left(X \leftarrow u_{t} X\right) \hat{\phi}(\mathbf{x}) U_{t}\left(u_{t} X \leftarrow X\right)\right] f .
\end{aligned}
$$

Следовательно, можно записать

$$
\hat{\varphi}_{h}(x) K_{X}^{h} f \simeq K_{X}^{h}\left[\frac{\Phi(x \mid X)}{\sqrt{h}}+\hat{\phi}(x \mid X)\right] f
$$

где $\Phi(x \mid X)$ - числовая функция, зависяшая от пространственно-временного аргумента $x$ и классического состояния $X, \hat{\phi}(x \mid X)$ - операторная обобшенная функция в $\mathcal{F}_{X}$. При этом справедливы уравнения

$$
\partial_{\mu} \partial^{\mu} \Phi(x \mid X)+V^{\prime}(\Phi(x \mid X))=0, \quad \partial_{\mu} \partial^{\mu} \hat{\phi}(x \mid X)+V^{\prime \prime}(\Phi(x \mid X)) \hat{\phi}(x \mid X)=0,
$$

которые можно получить, например, из гейзенберговских уравнений движения для $\hat{\varphi}_{h}(x)$.

Рассмотрим свойство релятивистской ковариантности полей в квазиклассическом приближении. В “точной” теории для преобразования Пуанкаре $g=(a, \Lambda)$ вида $x^{\prime}=$ $\Lambda x+a$ справедливо свойство

$$
\mathcal{U}_{g^{-1}}^{h} \hat{\varphi}_{h}(x) \mathcal{U}_{g}^{h}=\hat{\varphi}_{h}\left(w_{g} x\right), \quad w_{g} x=\Lambda^{-1}(x-a)
$$


Проводя выкладки, аналогичные (2.9), получаем отсюда соотношение

$$
\hat{\varphi}_{h}\left(w_{g} x\right) K_{X}^{h} f \simeq K_{X}^{h}\left[\frac{\Phi\left(w_{g} x \mid X\right)}{\sqrt{h}}+\hat{\phi}\left(w_{g} x \mid X\right)\right] f .
$$

Приходим к следуюшим квазиклассическим аналогам полевых аксиом.

A3 (оператор поля). Каждому классическому состоянию $X \in \mathcal{X}$ соответствуют числовая функиия $\Phi(x \mid X)$ и операторная обобщенная функиия $\hat{\phi}(x \mid X)$, действующая в пространстве $\mathcal{F}_{X}$.

А4 (релятивистская инвариантность полей). Справедливы свойства:

$$
\begin{aligned}
\Phi\left(w_{g} x \mid X\right) & =\Phi\left(x \mid u_{g} X\right) ; \\
\hat{\phi}\left(w_{g} x \mid X\right) & =U_{g^{-1}}\left(X \leftarrow u_{g} X\right) \hat{\phi}\left(x \mid u_{g} X\right) U_{g}\left(u_{g} X \leftarrow X\right) .
\end{aligned}
$$

2.3. На квазиклассическом расслоении можно ввести и структуры [15], связанные непосредственно с квазиклассическим приближением, а не с квантовыми полями и релятивистской инвариантностью.

Заметим, что при сдвиге классического состояния $X \in \mathcal{X}$ на малую величину $h \delta X$ порядка $h$ состояние $K_{X}^{h} f$ в главном порядке по $h$ умножается на число, которое будем обозначать как $e^{-i \omega_{X}[\delta X]}$ :

$$
K_{X+\delta X}^{h} f \simeq e^{-i \omega_{X}[\delta X]} K_{X}^{h} f
$$

при сдвиге же на величину порядка $\sqrt{h}$ преобразование канонического оператора является более сложным:

$$
K_{X+\sqrt{h} \delta X}^{h} f \simeq \operatorname{const} K_{X}^{h} e^{i \Omega_{X}[\delta X]} f,
$$

где const - числовой множитель, $\Omega_{X}[\delta X]$ - некоторый эрмитов оператор. При этом $\omega_{X}$ и $\Omega_{X}$ являются соответственно числовой и операторнозначной дифференциальной 1-формами на $\mathcal{X}$. Можно показать [15], [16], что при достаточно общих предположениях коммутатор операторов $\Omega_{X}[\delta X]$ является оператором умножения на число и связан с 2-формой $d \omega_{X}-$ дифференциалом 1-формы $\omega_{X}$ :

$$
\left[\Omega_{X}[\delta X] ; \Omega_{X}\left[\delta X^{\prime}\right]\right]=-i d \omega_{X}\left(\delta X, \delta X^{\prime}\right)
$$

Для рассматриваемого случая

$$
\begin{aligned}
& \omega_{X}[\delta X]=\int d \mathbf{x} \Pi(\mathbf{x}) \delta \Phi(\mathbf{x})-\delta S \\
& \Omega_{X}[\delta X]=\int d \mathbf{x}[\delta \Pi(\mathbf{x}) \hat{\varphi}(\mathbf{x})-\delta \Phi(\mathbf{x}) \hat{\pi}(\mathbf{x})] .
\end{aligned}
$$

Важным свойством 1-форм $\omega$ и $\Omega$ является их инвариантность относительно эволюции, которая связана с тем, что векторы $K_{X^{t}+h \delta X^{t}}^{h} f^{t}$ и $K_{X^{t}+\sqrt{h} \delta X^{t}}^{h} f^{t}$ должны приближенно удовлетворять уравнению Шредингера, если $\delta X^{t}$ - решение уравнения в вариациях, отвечающего классическому уравнению эволюции для $X^{t}$. Аналогичное свойство справедливо и для преобразований Пуанкаре.

3 Теоретическая и математическая физика, т. 144, № 3, 2005 г. 
Приходим к следуюшим аксиомам.

A5. На классическом пространстве $\mathcal{X}$ определень 1-формы $\omega$ (со значениями в $\mathbb{R})$ и $\Omega$ (со значениями в пространстве операторов в $\left.\mathcal{F}_{X}\right)$. При этом справедливо коммутационное соотношение (2.15).

А6 (релятивистская инвариантность 1-форм). Пусть $u_{g}(X+\delta X) \simeq X^{\prime}+\delta X^{\prime}$. Тогда

$$
\begin{aligned}
\omega_{X^{\prime}}\left[\delta X^{\prime}\right] & =\omega_{X}[\delta X] \\
\Omega_{X^{\prime}}\left[\delta X^{\prime}\right] U_{g}\left(X^{\prime} \leftarrow X\right) & =U_{g}\left(X^{\prime} \leftarrow X\right) \Omega_{X}[\delta X]
\end{aligned}
$$

2.4. Разработанная Масловым теория лагранжевых многообразий с комплексным ростком [1], [2] является обобщением теории комплексного ростка Маслова в точке. Одна из возможных формулировок заключается в следуюшем [5], [15], [17]. Рассматриваются состояния, являюшиеся суперпозициями состояний $(2.2)$ :

$$
\int d \alpha K_{X(\alpha)}^{h} f(\alpha)
$$

здесь $\alpha=\left(\alpha_{1}, \ldots, \alpha_{k}\right),(X(\alpha), f(\alpha))-k$-мерная поверхность в пространстве квазиклассического расслоения. Оказывается, что состояние (2.20) имеет экспоненциально малую при $h \rightarrow 0$ норму всегда, кроме специального случая

$$
\omega_{X}\left[\frac{\partial X}{\partial \alpha_{a}}\right]=0
$$

и только этот случай рассматривается. При выполнении условия (2.21) ("условия изотропоности Маслова") квадрат нормы вектора (2.20) равен

$$
h^{k / 2} \int d \alpha\left(f(\alpha), \prod_{a} 2 \pi \delta\left(\Omega_{X}\left[\frac{\partial X}{\partial \alpha_{a}}\right]\right) f(\alpha)\right) .
$$

Следовательно, для нормировки на единицу состояние (2.20) следует разделить на $h^{k / 4}$. Входящие в (2.22) дельта-функции коммутируют ввиду свойств (2.15) и (2.21).

Отметим, что формула (2.22) напоминает скалярное произведение, используемое в одном из способов квантования систем со связями [18].

При преобразованиях Пуанкаре состояние (2.20) переходит в

$$
h^{-k / 4} \int d \alpha K_{X(\alpha)}^{h} f(\alpha) \mapsto h^{-k / 4} \int d \alpha K_{u_{g} X(\alpha)}^{h} U_{g}\left(u_{g} X(\alpha) \leftarrow X(\alpha)\right) f(\alpha) .
$$

Инвариантность 1-форм $\omega$ и $\Omega$ является важным условием самосогласованности теории лагранжевых многообразий с комплексным ростком, поскольку условие изотропности Маслова (2.21) и скалярное произведение (2.22) должны сохраняться при преобразованиях Пуанкаре. 
2.5. Развиваемая ниже ковариантная формулировка квантово-полевой теории комплексного ростка Маслова во многом напоминает квазиклассическую механику систем со связями (калибровочных теорий) [19]. Ее специфика заключается в том, что некоторые классические состояния объявляются эквивалентными друг другу. Для любой пары классически эквивалентных состояний $X \sim X^{\prime}$ определен изоморфизм слоев $V\left(X^{\prime} \leftarrow\right.$ $X): \mathcal{F}_{X} \rightarrow \mathcal{F}_{X^{\prime}}$, удовлетворяюший свойству

$$
V\left(X^{\prime \prime} \leftarrow X\right)=X\left(X^{\prime \prime} \leftarrow X^{\prime}\right) V\left(X^{\prime} \leftarrow X\right) .
$$

Точки на квазиклассическом расслоении $(X, f) \sim\left(X^{\prime}, V\left(X^{\prime} \leftarrow X\right) f\right)$ называются калибровочно-эквивалентными.

Квазиклассические преобразования Пуанкаре, 1-формы $\omega$ и $\Omega$, функции $\Phi$ и $\hat{\phi}$ должны сохранять отношение калибровочной эквивалентности. Следовательно,

$$
\begin{aligned}
& \begin{array}{c}
u_{g} X \sim u_{g} X^{\prime}, \\
V\left(u_{g} X^{\prime} \leftarrow u_{g} X\right) U_{g}\left(u_{g} X \leftarrow X\right)=U_{g}\left(u_{g} X^{\prime} \leftarrow X^{\prime}\right) V\left(X^{\prime} \leftarrow X\right) \quad \text { при } \quad X \sim X^{\prime} ;
\end{array} \\
& \Phi(x \mid X)=\Phi\left(x \mid X^{\prime}\right), \\
& V\left(X^{\prime} \leftarrow X\right) \hat{\phi}(x \mid X)=\hat{\phi}\left(x \mid X^{\prime}\right) V\left(X^{\prime} \leftarrow X\right) \quad \text { при } \quad X \sim X^{\prime} \text {; } \\
& \omega_{X}[\delta X]=\omega_{X^{\prime}}\left[\delta X^{\prime}\right] \\
& V\left(X^{\prime} \leftarrow X\right) \Omega_{X}[\delta X]=\Omega_{X^{\prime}}\left[\delta X^{\prime}\right] V\left(X^{\prime} \leftarrow X\right) \\
& \text { при } \begin{aligned}
X & \sim X^{\prime}, \\
X+\delta X & \sim X^{\prime}+\delta X^{\prime} .
\end{aligned}
\end{aligned}
$$

В частности, при бесконечно малом калибровочном преобразовании $X+\delta X \sim X$ должны выполняться соотношения $\omega_{X}[\delta X]=0, \Omega_{X}[\delta X]=0$. Условия $(2.23)-(2.26)$ связаны с внутренней самосогласованностью квазиклассической теории.

\section{3. ОБ АКСИОМАТИЧЕСКОМ ПОДХОДЕ В КВАЗИКЛАССИЧЕСКОЙ ТЕОРИИ ПОЛЯ}

3.1. Квазиклассические состояния. При аксиоматическом построении ковариантной квазиклассической теории поля мы не будем пользоваться какими-либо эволюционными уравнениями движения, а ограничимся обычными предположениями аксиоматического подхода [8] о пуанкаре-ковариантности теории, сушествовании вакуумного вектора $|0\rangle$, пуанкаре-инвариантности оператора поля $\hat{\varphi}_{h}(x)$ и хронологической экспоненты

$$
T_{J}^{h}=T \exp \left\{\frac{i}{\sqrt{h}} \int d x J(x) \hat{\varphi}_{h}(x)\right\} .
$$

Остальные предположения будем оговаривать особо.

ПРЕДПОЛОЖЕНИЕ 1. Гильбертовъ пространства $\mathcal{H}^{h}$ совпадают, а операторы $\hat{\varphi}_{h}(x)$ и $\mathcal{U}_{g}^{h}$ разлагаются в асимптотические рядъ по $\sqrt{h}$.

Это предположение фактически используется, например, при построении формальной теории возмущений при использовании асимптотического in-представления в $S$-матричном подходе.

Обозначим эти операторы в главном порядке по $h$ как $\hat{\varphi}_{0}(x)$ и $U_{g}$, а соответствующее гильбертово пространство - как $\mathcal{H}$. 
Будем рассматривать состояния вида (1.3)

$$
\Psi=e^{(i / h) \bar{S}} T_{J}^{h} \bar{f} \equiv \bar{K} \frac{h}{S}, J \bar{f},
$$

где $\bar{S}$ - вешественное число, а $J(x)$ имеет компактный носитель $\operatorname{supp} J$ в области $x^{0} \in$ $\left[T_{-}, T_{+}\right]$.

Для дальнейшего введем следуюшее обозначение. Для точек $x$ и $y$ пространства Минковского будем писать $x<y, x>y$ и $x \sim y$, если $x^{0}>y^{0}, x^{0}<y^{0}$ и $(x-y)^{2}<0$, соответственно; $x \gtrsim y$, если $x>y$ или $x \sim y$. Аналогичные обозначения введем и для подмножеств: например, $x \gtrsim \operatorname{supp} J$, если $x \gtrsim y$ при всех $y \in \operatorname{supp} J$.

Отождествим состояния (3.2) с точками на квазиклассическом расслоении, базой которого является множество наборов $\bar{X}=\{\bar{S}, J(x)\}$, а слоями - гильбертовы пространства $\mathcal{H}$. Исследуем сформулированные в предыдущем разделе аксиомы квазиклассической теории поля.

Аксиома А2. Поскольку $\mathcal{U}_{g}^{h} T_{J}^{h} \bar{f}=T_{u_{g} J}^{h} \mathcal{U}_{g}^{h} \bar{f} \simeq T_{u_{g} J}^{h} U_{g} \bar{f}$, где $u_{g} J(x)=J\left(w_{g} x\right), w_{g}$ имеет вид (2.11), группа Пуанкаре действует на квазиклассическом расслоении как $(\bar{S}, J, \bar{f}) \mapsto\left(\bar{S}, u_{g} J, U_{g} \bar{f}\right)$.

Аксиома А3. Исследуем оператор поля при $x>\operatorname{supp} J$. Можно записать

$$
\hat{\varphi}_{h}(x) T_{J}^{h}=\frac{\sqrt{h}}{i} \frac{\delta T_{J}^{h}}{\delta J(x)}
$$

и

$$
\hat{\varphi}_{h}(x) T_{J}^{h} \bar{f}=T_{J}^{h} \frac{1}{\sqrt{h}} R(x \mid J) \bar{f},
$$

где

$$
R(x \mid J)=-i h\left(T_{J}^{h}\right)+\frac{\delta T_{J}^{h}}{\delta J(x)}
$$

- известная в аксиоматической теории поля $R$-функция ЛСЦ [8], [9]. Наложим на нее следуюшее условие.

ПреДПОЛОЖенИЕ 2. R-функция разлагается в асимптотический ряд по $\sqrt{h}$ :

$$
R(x \mid J)=\bar{\Phi}(x \mid J)+\sqrt{h} R^{(1)}(x \mid J)+\cdots,
$$

и в нулевом порядке теории возмущений является оператором умножения на числовую функцию $\bar{\Phi}(x \mid J)$.

При данном предположении числовая функция $\Phi(x \mid \bar{X})$ и операторная обобщенная функция $\hat{\phi}(x \mid \bar{X})$ при $x>\operatorname{supp} J$ имеют вид

$$
\Phi(x \mid \bar{X})=\bar{\Phi}(x \mid J), \quad \hat{\phi}(x \mid \bar{X})=R^{(1)}(x \mid J), \quad x>\operatorname{supp} J .
$$


Аксиома А5. 1-формы $\omega$ и $\Omega$ можно ввести из определения [16]

$$
i h \delta\left\{e^{(i / h) \bar{S}_{J}} T_{J}^{h} \simeq T_{J}^{h}\left\{\bar{\omega}_{\bar{X}}[\delta \bar{X}]-\sqrt{h} \bar{\Omega}_{\bar{X}}[\delta \bar{X}]\right\}\right.
$$

отсюда получаем

$$
\bar{\omega}_{\bar{X}}[\delta \bar{X}]-\sqrt{h} \bar{\Omega}_{\bar{X}}[\delta \bar{X}] \simeq-\int d x R(x \mid J) \delta J(x)-\delta \bar{S}
$$

$$
\begin{aligned}
& \bar{\omega}_{\bar{X}}[\delta \bar{X}]=-\int d x \bar{\Phi}(x \mid J) \delta J(x)-\delta \bar{S}, \\
& \bar{\Omega}_{\bar{X}}[\delta \bar{X}]=\int d x R^{(1)}(x \mid J) \delta J(x) .
\end{aligned}
$$

Тем самым основные объекты квазиклассической теории поля представляются через $R$-функции ЛСЦ. Следующие их свойства хорошо известны [8]

УТВЕРЖДЕНИЕ 1. 1. $R(x \mid J)$ - эрмитова операторная обобщенная функиия, зависящая только от $J(y)$ в предшествующие моменты времени (микропричинность Боголюбова); при $x<\operatorname{supp} J$ она имеет вид $R(x \mid J)=\hat{\varphi}_{h}(x) \sqrt{h}$.

2. R-Функиия релятивистски-ковариантна: $\mathcal{U}_{g^{-1}}^{h} R\left(x \mid u_{g} J\right) \mathcal{U}_{g}^{h}=R\left(w_{g} x \mid J\right)$.

3. Справедливо коммутационное свойство

$$
[R(x \mid J) ; R(y \mid J)]=-i h\left(\frac{\delta R(x \mid J)}{\delta J(y)}-\frac{\delta R(y \mid J)}{\delta J(x)}\right) .
$$

СлЕДСТВИЕ 1. 1. Функиия $\bar{\Phi}(x \mid J)$ вещественна, обращается в нуль при $x<$ supp $J$, зависит только от $J(y)$ при $y^{0}<x^{0}$ и удовлетворяет свойству релятивистской инвариантности $\bar{\Phi}\left(w_{g} x \mid J\right)=\bar{\Phi}\left(x \mid u_{g} J\right)$.

2. Операторная функция $R^{(1)}(x \mid J)$ эрмитова, совпадает $c \hat{\varphi}_{0}(x)$ при $x<$ $\operatorname{supp} J$, зависит только от $J(y)$ при $y^{0}<x^{0}$, удовлетворяет свойству релятивистской инвариантности

$$
U_{g^{-1}} R^{(1)}\left(x \mid u_{g} J\right) U_{g}=R^{(1)}\left(w_{g} x \mid J\right)
$$

и коммутационному соотношению

$$
\left[R^{(1)}(x \mid J) ; R^{(1)}(y \mid J)\right]=-i\left(\frac{\delta \bar{\Phi}(x \mid J)}{\delta J(y)}-\frac{\delta \bar{\Phi}(y \mid J)}{\delta J(x)}\right) .
$$

Таким образом, релятивистская инвариантность полей и 1-форм вытекает из общих свойств $R$-функций ЛСЦ. Коммутационное соотношение (3.9) совпадает с (2.15).

3.2. Эквивалентность квазиклассических состояний. Как сказано во введении, в ковариантном подходе квазиклассические состояния могут быть эквивалентны друг другу. Будем говорить, что $J \sim 0$, если

$$
T_{J}^{h} \bar{f} \simeq e^{(i / h) \bar{I}_{J}} W_{J} \bar{f}
$$

для некоторого числа $\bar{I}_{J}$ и оператора $W_{J}$, допускающего разложение в асимптотический ряд по $\sqrt{h}$.

Обозначим через $W_{J}^{0}$ оператор $W_{J}$ в главном порядке по $\sqrt{h}$. Исследуем свойства $\bar{I}_{J}$ и $W_{J}$. 
УТВЕРЖДЕНИЕ 2. 1. Оператор $W_{J}$ унитарен.

2. Пусть $J \sim 0$. Тогда $u_{g} J \sim 0$, причем

$$
\bar{I}_{u_{g} J}=\bar{I}_{J}, \quad U_{g} W_{J}^{0} U_{g}^{-1}=W_{u_{g} J}^{0} .
$$

3. При $x \gtrsim \operatorname{supp} J$ справедливы свойства

$$
\bar{\Phi}(x \mid J)=0, \quad R^{(1)}(x \mid J)=\left(W_{J}^{0}\right)^{+} \hat{\varphi}_{0}(x) W_{J}^{0} .
$$

Первое свойство следует из унитарности оператора $T_{J}^{h}$, второе получается применением оператора $\mathcal{U}_{g}^{h}$ к соотношению (3.10), свойства же (3.12) вытекают из соотношения $T_{J}^{h} R(x \mid J) \bar{f}=\sqrt{h} \hat{\varphi}_{h}(x) T_{J}^{h} \bar{f}, x \gtrsim \operatorname{supp} J$, которое приводится к виду $R(x \mid J)=$ $\sqrt{h} W_{J}^{+} \hat{\varphi}(x) W_{J}$ и разлагается в ряд теории возмушений.

УТВЕРЖДЕНИЕ 3. При $J \sim 0, J+\delta J \sim 0$

$$
\delta \bar{I}=\int d x \bar{\Phi}(x \mid J) \delta J(x), \quad \int d x R^{(1)}(x \mid J) \delta J(x)=0 .
$$

Для доказательства рассмотрим вариацию соотношения (3.10):

$$
\delta T_{J}^{h} \cdot \bar{f} \simeq \frac{i}{h} \delta \bar{I}_{J} T_{J}^{h} \bar{f}+T_{J}^{h} W_{J}^{+} \delta W_{J} \bar{f} .
$$

Следовательно,

$$
-i h\left(T_{J}^{h}\right)^{+} \delta T_{J}^{h}=\delta \bar{I}_{J}-i h W_{J}^{+} \delta W_{J} .
$$

С другой стороны, согласно (3.4)

$$
-i h\left(T_{J}^{h}\right)^{+} \delta T_{J}^{h}=\int d x R(x \mid J) \delta J(x)
$$

Сравнивая (3.14) и (3.15), получаем (3.13).

Следуюшее утверждение выражает принцип причинности для $W_{J}^{0}$.

УТВЕРЖДЕНИЕ 4. Пусть $J+\Delta J_{2} \sim 0, J+\Delta J_{1}+\Delta J_{2} \sim 0, \operatorname{supp} \Delta J_{2} \gtrsim \operatorname{supp} \Delta J_{1}$. Тогда оператор $\left(W_{J+\Delta J_{2}}^{0}\right)^{+} W_{J+\Delta J_{1}+\Delta J_{2}}^{0}$ и число $-\bar{I}_{J+\Delta J_{2}}+\bar{I}_{J+\Delta J_{1}+\Delta J_{2}}$ не завиcят от $\Delta J_{2}$.

Для доказательства достаточно рассмотреть оператор

$$
\left(T_{J+\Delta J_{2}}^{h}\right)^{+} T_{J+\Delta J_{1}+\Delta J_{2}}^{h} \simeq e^{(i / h)\left[-\bar{I}_{J+\Delta J_{2}}+\bar{I}_{J+\Delta J_{1}+\Delta J_{2}}\right]}\left(W_{J+\Delta J_{2}}^{0}\right)^{+} W_{J+\Delta J_{1}+\Delta J_{2}}^{0},
$$

который не зависит от $\Delta J_{2}$.

Введем теперь отношение эквивалентности на квазиклассическом расслоении. Будем говорить, что $J_{1} \sim J_{2}$, если для некоторого источника $J_{+}$с носителем в будушем от источников $J_{1}$ и $J_{2}\left(\operatorname{supp} J_{+} \gtrsim \operatorname{supp} J_{1} \cup \operatorname{supp} J_{2}\right)$ справедливы свойства $J_{1}+J_{+} \sim 0$, $J_{2}+J_{+} \sim 0$. 
УТВЕРЖДЕНИЕ 5. Если $J_{1} \sim J_{2}$ и $J_{+}^{\prime}-$ функиия, удовлетворяющая свойствам $\operatorname{supp} J_{+}^{\prime} \gtrsim \operatorname{supp} J_{1} \cup \operatorname{supp} J_{2} u J_{1}+J_{+}^{\prime} \sim 0$, mo $u J_{2}+J_{+}^{\prime} \sim 0$.

Для доказательства достаточно воспользоваться свойством

$$
T_{J_{2}+J_{+}^{\prime}}^{h}=T_{J_{1}+J_{+}^{\prime}}^{h}\left(T_{J_{2}+J_{+}}^{h}\right)^{+} T_{J_{1}+J_{+}}^{h} .
$$

СлЕДСТвИЕ 2. При $J_{1} \sim J_{2}$ и $J_{2} \sim J_{3}$ справедливо свойство $J_{1} \sim J_{3}$.

УТВЕРЖДЕнИЕ 6 . Пусть $J_{1} \sim J_{2}$. Тогда свойство

$$
e^{(i / h) \bar{S}_{1}} T_{J_{1}}^{h} \bar{f}_{1} \simeq e^{(i / h) \bar{S}_{2}} T_{J_{2}}^{h} \bar{f}_{2}
$$

справедливо тогда и только тогда, когда

$$
\bar{S}_{1}+\bar{I}_{J_{1}+J_{+}}=\bar{S}_{2}+\bar{I}_{J_{2}+J_{+}}, \quad \bar{f}_{2}=\left(W_{J_{2}+J_{+}}^{0}\right)^{+} W_{J_{1}+J_{+}}^{0} \bar{f}_{1} .
$$

Для доказательства достаточно подействовать оператором $T_{J_{+}}^{h}$ на левую и правую части соотношения (3.16).

Назовем два квазиклассических состояния $\left(\bar{X}_{1}, \bar{f}_{1}\right) \sim\left(\bar{X}_{2}, \bar{f}_{2}\right)$ эквивалентными при условии (3.17). Во введенных выше обозначениях

$$
V\left(\bar{X}_{2} \leftarrow \bar{X}_{1}\right)=\left(W_{J_{2}+J_{+}}^{0}\right)^{+} W_{J_{1}+J_{+}}^{0} \equiv V\left(J_{2} \leftarrow J_{1}\right) .
$$

Из свойства причинности (утверждение 4) вытекает, что определение (3.18) не зависит от выбора источника $J_{+}$.

Проверим свойства (2.23)-(2.26). Свойство (2.23) очевидно. Свойство (2.24) вытекает из релятивистской инвариантности (утверждение 2). Свойство (2.26) для $\omega$ проверяется следуюшим образом. Пусть $\left(\bar{S}_{1}, J_{1}\right) \sim\left(\bar{S}_{2}, J_{2}\right),\left(\bar{S}_{1}+\delta \bar{S}_{1}, J_{1}+\delta J_{1}\right) \sim\left(\bar{S}_{2}+\right.$ $\left.\delta \bar{S}_{2}, J_{2}+\delta J_{2}\right)$. Согласно (3.17) это означает, что

$$
\delta \bar{S}_{1}+\int d x \bar{\Phi}\left(x \mid J_{1}\right) \delta J_{1}=\delta \bar{S}_{2}+\int d x \bar{\Phi}\left(x \mid J_{2}\right) \delta J_{2},
$$

или $\omega_{\bar{X}_{1}}\left[\delta \bar{X}_{1}\right] \omega_{\bar{X}_{2}}\left[\delta \bar{X}_{2}\right]$.

Далее, подействуем на равенство (3.16) оператором $\hat{\varphi}_{h}(x)$. Получим

$$
e^{(i / h) \bar{S}_{1}} T_{J_{1}}^{h} R\left(x \mid J_{1}\right) \bar{f}_{1}=e^{(i / h) \bar{S}_{2}} T_{J_{2}}^{h} R\left(x \mid J_{2}\right) \bar{f}_{2} .
$$

Отсюда

$$
R\left(x \mid J_{2}\right) V\left(J_{2} \leftarrow J_{1}\right)=V\left(J_{2} \leftarrow J_{1}\right) R\left(x \mid J_{1}\right) .
$$

Разлагая данное соотношение по теории возмушений, при $x \gtrsim \operatorname{supp} J_{1} \cup \operatorname{supp} J_{2}$ находим

$$
\bar{\Phi}\left(x \mid J_{1}\right)=\bar{\Phi}\left(x \mid J_{2}\right), \quad R^{(1)}\left(x \mid J_{2}\right) V\left(J_{2} \leftarrow J_{1}\right)=V\left(J_{2} \leftarrow J_{1}\right) R^{(1)}\left(x \mid J_{1}\right) .
$$

Следовательно, свойство (2.25) проверено при $x \gtrsim \operatorname{supp} J$ (при тех $x$, при которых были определены $\Phi$ и $\hat{\phi}$ выше). Свойства (2.25) можно использовать для доопределения $\Phi$ и $\hat{\phi}$ и при остальных $x$ : для этого нужно подобрать источник $J^{\prime} \sim J$, удовлетворяюший условию $\operatorname{supp} J \lesssim x$, и положить

$$
\Phi(x \mid \bar{X}) \equiv \bar{\Phi}\left(x \mid J^{\prime}\right) ; \quad \hat{\phi}(x \mid \bar{X}) \equiv V\left(J \leftarrow J^{\prime}\right) R^{(1)}\left(x \mid J^{\prime}\right) V\left(J^{\prime} \leftarrow J\right) .
$$

Свойства (2.25) при таком доопределении останутся справедливыми.

Проверим свойство (2.26) для $\Omega$. 
УТВЕРЖДЕНИЕ 7. Пусть $J_{1} \sim J_{2}, J_{1}+\delta J_{1} \sim J_{2}+\delta J_{2}$. Тогда

$$
\int d x R^{(1)}\left(x \mid J_{2}\right) \delta J_{2} V\left(J_{2} \leftarrow J_{1}\right)=V\left(J_{2} \leftarrow J_{1}\right) \int d x R^{(1)}\left(x \mid J_{1}\right) \delta J_{1}(x) .
$$

Для доказательства введем операторную функцию

$$
\widetilde{R}(x \mid J)=T_{J}^{h} R(x \mid J)\left(T_{J}^{h}\right)^{+} .
$$

Она зависит только от $J(y)$ в последующие моменты времени, удовлетворяет граничному условию $\widetilde{R}(x \mid J)=\hat{\varphi}(x) \sqrt{h}$ при $x \gtrsim \operatorname{supp} J$. При $J \sim 0 \widetilde{R}$-функция имеет вид $\widetilde{R}(x \mid J)=W_{J} R(x \mid J) W_{J}^{+}$, разлагается в асимптотический ряд

$$
\widetilde{R}(x \mid J)=\widetilde{\Phi}(x \mid J)+\sqrt{h} \widetilde{R}^{(1)}(x \mid J)+\cdots,
$$

причем

$$
\widetilde{\Phi}(x \mid J)=\Phi(x \mid J), \quad \widetilde{R}^{(1)}(x \mid J)=W_{J}^{0} R^{(1)}(x \mid J)\left(W_{J}^{0}\right)^{+} .
$$

Пусть $J_{1} \sim J_{2}$ и $J_{1}+\delta J_{1} \sim J_{2}+\delta J_{2}$. Это означает, что $J_{1}+J_{+} \sim 0, J_{2}+J_{+} \sim 0$, $J_{1}+\delta J_{1}+J_{+}+\delta J_{+} \sim 0, J_{2}+\delta J_{2}+J_{+}+\delta J_{+} \sim 0$ при $\operatorname{supp} J_{+} \cup \operatorname{supp} \delta J_{+} \gtrsim \operatorname{supp} J_{1} \cup$ $\operatorname{supp} J_{2} \cup \operatorname{supp} \delta J_{1} \cup \operatorname{supp} \delta J_{2}$. Как вытекает из (3.13), (3.18), (3.23), свойство (3.21) равносильно следуюшему:

$$
\begin{aligned}
& \left(W_{J_{2}+J_{+}}^{0}\right)^{+} \int d x \widetilde{R}^{(1)}\left(x \mid J_{2}+J_{+}\right) \delta J_{+}(x) W_{J_{1}+J_{+}}^{0}= \\
& =\left(W_{J_{2}+J_{+}}^{0}\right)^{+} \int d x \widetilde{R}^{(1)}\left(x \mid J_{1}+J_{+}\right) \delta J_{+}(x) W_{J_{1}+J_{+}}^{0}
\end{aligned}
$$

которое следует из условия причинности для $\widetilde{R}^{(1)}$ :

$$
\widetilde{R}^{(1)}\left(x \mid J_{1}+J_{+}\right)=\widetilde{R}^{(1)}\left(x \mid J_{2}+J_{+}\right)=\widetilde{R}^{(1)}\left(x \mid J_{+}\right) .
$$

Утверждение доказано.

3.3. О квазиклассическом ковариантном квантовании полей. Развиваемая аксиоматика квазиклассической теории поля может быть положена в основу квазиклассического квантования полей. Чтобы получить “из первых принципов" классические уравнения поля, наложим следуюшее условие.

ПРЕДПОЛОЖЕНИЕ 3 . Для любой полевой конфигурации $\Phi(x)$ с компактныцм носителем можно однозначно подобрать источник $J \sim 0$ (обозначим его как $J=$ $J(x \mid \Phi))$, порождающий конфигурацию $\Phi: \Phi(x)=\bar{\Phi}(x \mid J)$. Он удовлетворяет условию локальности:

$$
\frac{\delta J(x \mid \Phi)}{\delta \Phi(y)}=0, \quad y \neq x .
$$

Из данного предположения и формулы (3.13) вытекает 
УТВЕРЖДЕНИЕ 8. 1. Функиионал $I[\Phi]=\bar{I}_{J_{\Phi}}-\int d x J(x) \Phi(x)$ удовлетворяет свойству локальности

$$
I[\Phi]=\int d x \mathcal{L}\left(\Phi(x), \partial_{\mu} \Phi(x), \ldots, \partial_{\mu_{1}} \ldots \partial \mu_{n} \Phi(x)\right)
$$

и принципу стационарного действия

$$
\frac{\delta I[\bar{\Phi}]}{\delta \Phi(x)}=-J(x)
$$

2. Операторная обобщенная функиия $R^{(1)}(x \mid J)$ удовлетворяет уравнению

$$
\int d y \frac{\delta^{2} I[\bar{\Phi}]}{\delta \Phi(x) \delta \Phi(y)} R^{(1)}(y \mid J)=0 .
$$

3. Обозначим через $D \frac{\text { ret }}{\Phi}(x, y)$ запаздьвающую функиию Грина задачи

$$
\int d y \frac{\delta^{2} I[\bar{\Phi}]}{\delta \Phi(x) \delta \Phi(y)} \delta \Phi(y)=-\delta J(x),\left.\quad \delta \Phi\right|_{x^{0}<T_{-}}=0
$$

определяемую из соотношения $\delta \Phi(x)=\int d y D_{\bar{\Phi}}^{\text {ret }}(x, y) \delta J(y)$. Тогда

$$
\left[R^{(1)}(x \mid J) ; R^{(1)}(y \mid J)\right]=-i\left(D \bar{\Phi}^{\text {ret }}(x, y)-D \bar{\Phi}^{\text {ret }}(y, x)\right) .
$$

Свойство (3.26) является другой записью (3.13), поскольку

$$
\delta I=\delta \bar{I}-\int d x(\delta J \bar{\Phi}+J \delta \bar{\Phi})=-\int d x J \delta \bar{\Phi} .
$$

Свойство локальности $I$ вытекает из (3.25). Далее, пусть $\Phi$ и $\Phi+\delta \Phi-$ две полевые конфигурации с компактным носителем. Тогда для соответствующих источников $J, J+$ $\delta J$ имеем

$$
\delta J(y)=-\int d x \frac{\delta^{2} I[\bar{\Phi}]}{\delta \Phi(x) \delta \Phi(y)} \delta \Phi(x),
$$

и свойство (3.13) записывается как

$$
-\int d x d y R^{(1)}(y \mid J) \frac{\delta^{2} I[\bar{\Phi}]}{\delta \Phi(x) \delta \Phi(y)} \delta \Phi(x)=0,
$$

откуда получаем (3.27). Коммутационное соотношение (3.28) является другой записью (3.9). Утверждение доказано.

Из изложенного вытекает, что квазиклассическая теория в главном порядке по $h$ восстанавливается по функционалу действия $I[\Phi]$ без использования постулатов канонического квантования. Проясняется и физический смысл функционала действия: если $\Phi(x)$ - полевая конфигурация с компактным носителем, то ей однозначно сопоставляется источник $J \sim 0$, а соответствуюшая ему Т-экспонента $T_{J}^{h}$ равна произведению множителя $e^{(i / h) \bar{I}_{J}}$ на имеющий предел при $h \rightarrow 0$ оператор; величина $\bar{I}_{J}-\int d x J \Phi$ является классическим действием. 
При этом по известному $I[\Phi]$ строится классическое уравнение движения (3.26) для $\bar{\Phi}$, из которого с учетом граничного условия $\left.\Phi\right|_{x<\operatorname{supp} J}=0$ однозначно находится $\bar{\Phi}(x \mid J)$, а также уравнение движения (3.27) для операторов $R^{(1)}(x \mid J)$ и коммутационное соотношение (3.28) для этих операторов.

Для свободной классической теории поля

$$
I[\Phi]=\int d x\left[\frac{1}{2} \partial_{\mu} \Phi \partial^{\mu} \Phi-\frac{m^{2}}{2} \Phi^{2}\right]
$$

следовательно, классическое уравнение (3.26) имеет вид

$$
\left(\partial_{\mu} \partial^{\mu}+m^{2}\right) \bar{\Phi}(x)=J(x) .
$$

Оператор $R^{(1)}(x \mid J)$ удовлетворяет уравнению (3.27)

$$
\left(\partial_{\mu} \partial^{\mu}+m^{2}\right) R^{(1)}(x \mid J)=0
$$

Поскольку функция $D \frac{\text { ret }}{\Phi}(x, y)$ в данном случае совпадает с запаздывающей функцией Грина для уравнения $(3.30) D_{0}^{\text {ret }}(x, y)$, приводим коммутационное соотношение $(3.28)$ к виду

$$
\left[R^{(1)}(x \mid J), R^{(1)}(y \mid J)\right]=-i\left(D_{0}^{\mathrm{ret}}(x, y)-D_{0}^{\mathrm{ret}}(y, x)\right)=-i D_{0}(x, y) .
$$

Здесь $D_{0}(x, y)$ - перестановочная функция свободного поля.

Как вытекает из $(3.5)$, при $x \gtrsim \operatorname{supp~} J$ оператор поля $\hat{\phi}(x \mid \bar{X})=R^{(1)}(x \mid J)$ также удовлетворяет уравнению (3.31):

$$
\left(\partial_{\mu} \partial^{\mu}+m^{2}\right) \hat{\phi}(x \mid \bar{X})=0
$$

при $x \gtrsim \operatorname{supp} J$ и $y \gtrsim \operatorname{supp} J$ справедливо коммутационное соотношение

$$
[\hat{\phi}(x \mid \bar{X}), \hat{\phi}(y \mid \bar{X})]=-i D_{0}(x, y) .
$$

Ввиду формулы (3.20), продолжающей определение квазиклассического поля $\hat{\phi}(x \mid \bar{X})$ на остальные значения $x$, уравнения (3.33) и коммутационное соотношение (3.34) справедливы при всех $x$ и $y$.

Если принять во внимание также и аксиому о существовании и единственности вакуума, то соотношения (3.33) и (3.34) однозначно определяют, что $\hat{\phi}(x \mid X)$ - свободное скалярное поле $\hat{\varphi}_{0}(x)$. Оператор $U_{g}$ преобразования Пуанкаре однозначно определяется соотношениями

$$
U_{g^{-1}} \hat{\varphi}_{0}(x) U_{g}=\hat{\varphi}_{0}\left(w_{g} x\right), \quad U_{g}|0\rangle=|0\rangle .
$$

Поскольку $R^{(1)}(x \mid J)=\hat{\varphi}_{0}(x)$ как при $x \lesssim \operatorname{supp} J$, так и при $x \gtrsim \operatorname{supp} J$, из свойства (3.12) вытекает, что оператор $W_{J}^{0}$ является оператором умножения на числовой фазовый множитель.

Таким образом, квазиклассическое квантование свободной теории поля (с действием (3.29)) совпадает с другими, “точными” способами квантования. 
Для теории самодействующего скалярного поля с классическим действием

$$
I[\Phi]=\int d x\left[\frac{1}{2} \partial_{\mu} \Phi \partial^{\mu} \Phi-V(\Phi)\right],
$$

где $V(\Phi) \sim\left(m^{2} / 2\right) \Phi^{2}$ при $\Phi \rightarrow 0$, уравнения (3.26) и (3.27) запишутся как

$$
\begin{gathered}
\partial_{\mu} \partial^{\mu} \bar{\Phi}+V^{\prime}(\bar{\Phi})=J \\
\left(\partial_{\mu} \partial^{\mu}+V^{\prime \prime}(\bar{\Phi}(x))\right) R^{(1)}(x \mid J)=0
\end{gathered}
$$

$D \frac{\text { ret }}{\Phi}(x, y)$ оказывается запаздываюшей функцией Грина для уравнения

$$
\left(\partial_{\mu} \partial^{\mu}+V^{\prime \prime}(\bar{\Phi}(x))\right) \delta \Phi(x)=\delta J(x) .
$$

Ввиду граничного условия $R^{(1)}(x \mid J)=\hat{\varphi}_{0}(x)$ при $x \lesssim \operatorname{supp} J$ операторы $R^{(1)}(x \mid J)$ однозначно определяются из (3.35). Оператор $W_{J}^{0}$ однозначно определяется из соотношения (3.12) (при $x \gtrsim \operatorname{supp} J$ ) с точностью до фазового множителя $c_{J}$, который, как вытекает из свойства причинности, имеет вид

$$
\exp \left[i \int d x \mathcal{L}_{1}\left(\Phi(x), \partial_{\mu} \Phi(x), \ldots, \partial_{\mu_{1}} \ldots \partial_{\mu_{n}} \Phi(x)\right)\right]
$$

где $\mathcal{L}_{1}$ - однопетлевой контрчлен. При этом формально можно записать

$$
W_{J}^{0}=T \exp \left\{-\frac{i}{2} \int d x V^{\prime \prime}(\bar{\Phi}(x)) \hat{\varphi}_{0}^{2}(x)\right\}
$$

так как унитарный оператор $W_{J}^{0}$, связывающий поля при $x^{0} \rightarrow \pm \infty$, является матрицей рассеяния на внешнем классическом поле $\bar{\Phi}(x)$. Построение оператора $W_{J}^{0}$ эквивалентно анализу расходимостей в однопетлевом приближении.

Отметим, что введенное здесь соотношение (3.28), содержашее в правой части скобку Пайерлса [20] (см. также [21], [22]) классических полей в точках $x$ и $y$, рассматривалось в монографии [21] в качестве основного постулата квантования полей.

\section{4. ВЗАИМОСВЯЗЬ ГАМИЛЬТОНОВА И АКСИОМАТИЧЕСКОГО ПОДХОДОВ}

4.1. В двух предыдущих разделах рассмотрены гамильтонова и аксиоматическая формулировки квазиклассической теории поля. Исследуем их взаимосвязь.

Прежде всего покажем, что состояние (1.3), рассматриваемое в ковариантном подходе, может быть приведено к виду (2.2). Для этого заметим, что оператор

$$
T_{J}^{h}=T \exp \left[\frac{i}{\sqrt{h}} \int d x J(x) \hat{\varphi}_{h}(x)\right]
$$

связан с оператором эволюции $U_{J}\left(0, t_{-}\right)$для системы с гамильтонианом

$$
H_{J}(t)=H-\frac{1}{\sqrt{h}} \int d \mathbf{x} J(\mathbf{x}, t) \hat{\varphi}(\mathbf{x})
$$


стандартной формулой перехода в представление взаимодействия

$$
T_{J}^{h}=U_{J}\left(0, t_{-}\right) e^{-i H t_{-}}, \quad t_{-}<(\operatorname{supp} J)^{0}
$$

При этом

$$
e^{-i H t_{-}} \bar{f} \simeq e^{-i H_{0} t_{-}} \bar{f}
$$

где $H_{0}$ - гамильтониан свободного поля массы $m$. При квазиклассической эволюции с гамильтонианом $H_{J}$ квазиклассическое состояние (4.1) переходит в состояние (2.2); при этом для $S, \Pi, \Phi, f$ получаем систему уравнений, связанную с (2.3)-(2.5) заменой $V(\Phi) \Rightarrow V(\Phi)-J \Phi$. Если обозначить через $U_{2}\left(0, t_{-}\right)$оператор эволюции для уравнения (2.5), то окажется, что

$$
e^{(i / h) \bar{S}} T_{J}^{h} \bar{f} \simeq K_{S, \Pi, \Phi}^{h} f, \quad(\operatorname{supp} J)^{0}=\left[T_{-}, T_{+}\right] \subset(-\infty, 0),
$$

где в обозначениях предыдущего раздела

$$
\begin{gathered}
\Pi(\mathbf{x})=\dot{\bar{\Phi}}(x \mid J), \quad \Phi(\mathbf{x})=\bar{\Phi}(x \mid J), \quad S=\bar{S}+I_{-}[\Phi], \quad x^{0}=0 \\
I_{-}[\bar{\Phi}]=\int_{x^{0}<0} d x\left[\frac{1}{2} \partial_{\mu} \bar{\Phi} \partial^{\mu} \bar{\Phi}-V(\bar{\Phi})+J \bar{\Phi}\right] \\
f=\mathcal{V}_{\bar{X}} \bar{f}, \quad \mathcal{V}_{\bar{X}}=U_{2}\left(0, t_{-}\right) e^{-i H_{0} t_{-}}, \quad t_{-}<T_{-}
\end{gathered}
$$

Отсюда получаем свойство (1.4).

4.2. Исследуем взаимосвязь классических теорий поля в гамильтоновом и ковариантном подходах. В первом случае расширенным фазовым пространством (базой квазиклассического расслоения) является множество наборов $(S, \Pi(\mathbf{x}), \Phi(\mathbf{x}))$ с 1-формой действия

$$
\omega_{X}[\delta X]=\int d \mathbf{x} \Pi(\mathbf{x}) \delta \Phi(\mathbf{x})-\delta S .
$$

Во втором случае расширенное фазовое пространство можно рассматривать как поверхность в пространстве наборов $\{\bar{X}=(S, J(x), \bar{\Phi}(x))\}$, заданную уравнением $(3.26)$ :

$$
\partial_{\mu} \partial^{\mu} \bar{\Phi}(x)+V^{\prime}(\bar{\Phi}(x))=J(x),\left.\quad \Phi\right|_{x^{0}<T_{-}}=0
$$

1-форма действия записывается в виде

$$
\bar{\omega}_{X}[\delta \bar{X}]=-\int d x \bar{\Phi}(x) \delta J(x)-\delta \bar{S} .
$$

Соотношения (4.4) и (4.5) позволяют интерпретировать $J(x)$ и $\bar{\Phi}(x)$ как импульсы и координаты, соответственно, при этом соотношение (4.4) задает связи первого рода.

Эквивалентность гамильтоновой и ковариантной формулировок классической теории поля вытекает из соотношения $\bar{\omega}_{\bar{X}}[\delta \bar{X}]=\omega_{X}[\delta X]$, проверяемого непосредственным вычислением с использованием свойств (4.2) ввиду $\delta S=\delta \bar{S}+\delta I_{-}[\bar{\Phi}]$. Дифференциалы 
$d \omega$, задаюшие симплектическую структуру, также совпадают, что выражается равенством

$$
\int d \mathbf{x}\left[\delta \Pi_{1}(\mathbf{x}) \delta \Phi_{2}(\mathbf{x})-\delta \Pi_{2}(\mathbf{x}) \delta \Phi_{1}(\mathbf{x})\right]=\int d x\left[\delta J_{1}(x) \delta \Phi_{2}(x)-\delta J_{2}(x) \delta \Phi_{1}(x)\right] .
$$

Отметим, что генераторы преобразований Пуанкаре имеют в ковариантной формулировке простой вид:

$$
\begin{aligned}
\mathcal{P}^{\mu} & =\int d x J(x) \partial^{\mu} \bar{\Phi}(x), \\
\mathcal{M}^{\mu \nu} & =\int d x J(x)\left(x^{\mu} \partial^{\nu}-x^{\nu} \partial^{\mu}\right) \bar{\Phi}(x) ;
\end{aligned}
$$

с помощью уравнения (4.4) нетрудно привести эти генераторы к виду, используемому в гамильтоновой теории поля (в частности, $\mathcal{P}^{\mu}$ представляется в виде интеграла от тензора энергии-импульса $T^{\mu 0}$ по пространству). По аналогии с (4.6) можно записывать и генераторы любых преобразований, сохраняюших действие, которые совпадают с нетеровскими интегралами движения.

4.3. Исследуем теперь взаимосвязь других объектов квазиклассической теории в гамильтоновом и аксиоматическом подходах: квазиклассических преобразований Пуанкаре $U_{g}\left(u_{g} X \leftarrow X\right)$, полей $\hat{\Phi}(x \mid X), 1$-форм $\Omega$. Эквивалентность подходов означает, что должны выполняться соотношения

$$
\begin{aligned}
\mathcal{V}_{u_{g}} \bar{X}_{g} \bar{f} & =U_{g}\left(u_{g} X \leftarrow X\right) \mathcal{V}_{\bar{X}} \bar{f} \\
\mathcal{V}_{\bar{X}} R^{(1)}(x \mid J) \bar{f} & =\hat{\phi}(x \mid X) \mathcal{V}_{\bar{X}} \bar{f} ; \quad x \gtrsim \operatorname{supp} J \\
\mathcal{V}_{\bar{X}} \int d x R^{(1)}(x \mid J) \delta J(x) \bar{f} & =\int d \mathbf{x}\left[\delta \Pi(\mathbf{x}) \phi(\mathbf{x})-\delta \Phi(\mathbf{x}) \frac{1}{i} \frac{\delta}{\delta \phi(\mathbf{x})}\right] \mathcal{V}_{\bar{X}} \bar{f} .
\end{aligned}
$$

Соотношение (4.9) проверяется следуюшим образом. Прежде всего, учтем соотношение (4.4) и вытекающее из него свойство

$$
\partial_{\mu} \partial^{\mu} \delta \bar{\Phi}+V^{\prime \prime}(\bar{\Phi}) \delta \bar{\Phi}=\delta J,\left.\quad \delta \bar{\Phi}\right|_{x \lesssim \operatorname{supp} \delta J}=0,
$$

и запишем оператор в левой части в виде:

$$
\begin{aligned}
\int d x R^{(1)}(x \mid J) \delta J(x) & =\int d x R^{(1)}(x \mid J)\left(\partial_{\mu} \partial^{\mu} \delta \bar{\Phi}(x)+V^{\prime \prime}(\bar{\Phi}) \delta \bar{\Phi}(x)\right)= \\
& =\int d x\left[R^{(1)}(x \mid J) \partial_{\mu} \partial^{\mu} \delta \bar{\Phi}(x)-\delta \bar{\Phi}(x) \partial_{\mu} \partial^{\mu} R^{(1)}(x \mid J)\right]= \\
& =\int d \sigma^{\mu}\left[R^{(1)}(x \mid J) \partial^{\mu} \delta \bar{\Phi}(x)-\delta \bar{\Phi}(x) \partial^{\mu} R^{(1)}(x \mid J)\right] .
\end{aligned}
$$

В формуле (4.11) интегрирование осушествляется по любой пространственноподобной поверхности, удовлетворяюшей условию $x \gtrsim \operatorname{supp} J$. 
Обозначим через $\delta \Phi_{+}(x)$ решение задачи

$$
\left[\partial_{\mu} \partial^{\mu}+V^{\prime \prime}(\bar{\Phi}(x))\right] \delta \Phi_{+}=0,\left.\quad \delta \Phi_{+}\right|_{x^{0}>T_{+}}=\delta \bar{\Phi} ;
$$

тогда формулу (4.11) можно преобразовать к виду

$$
\int d x R^{(1)}(x \mid J) \delta J(x)=\int d \sigma^{\mu}\left[R^{(1)}(x \mid J) \partial^{\mu} \delta \Phi_{+}(x)-\delta \Phi_{+}(x) \partial^{\mu} R^{(1)}(x \mid J)\right],
$$

или

$$
\int d x R^{(1)}(x \mid J) \delta J(x)=\int d \sigma^{\mu}\left[\hat{\varphi}_{0}(x) \partial^{\mu} \delta \Phi_{+}(x)-\delta \Phi_{+}(x) \partial^{\mu} \hat{\varphi}_{0}(x)\right] .
$$

Пространственноподобная поверхность, по которой производится интегрирование, в формуле (4.13) произвольна, а в формуле (4.14) удовлетворяет условию $x \lesssim \operatorname{supp~} J$.

Заметим теперь, что оператор

$$
A(t)=\int_{x^{0}=t} d \mathbf{x}\left[\dot{\delta} \Phi_{+}(\mathbf{x}, t) \hat{\varphi}(\mathbf{x})-\delta \Phi_{+}(\mathbf{x}, t) \hat{\pi}(\mathbf{x})\right]
$$

коммутирует с оператором $i d / d t-H_{2}^{t}$ и поэтому переводит решения уравнения (2.5) в решения этого же уравнения. Следовательно, левая часть (4.9) равна

$$
\begin{aligned}
\mathcal{V}_{\bar{X}} e^{i H_{0} t_{-}} A\left(t_{-}\right) e^{-i H_{0} t_{-}} \bar{f} & =U_{2}\left(0, t_{-}\right) A\left(t_{-}\right) e^{-i H_{0} t_{-}} \bar{f}= \\
& =A(0) U_{2}\left(0, t_{-}\right) e^{-i H_{0} t_{-}} \bar{f}=A(0) \mathcal{V}_{\bar{X}} \bar{f}
\end{aligned}
$$

и совпадает с правой частью. Соотношение (4.9) проверено. Представляя его левую часть в виде интеграла (4.11) по поверхности $x^{0}=0$, приходим к соотношениям

$$
\mathcal{V}_{\bar{X}} R^{(1)}(x \mid J)=\hat{\phi}(x) \mathcal{V}_{\bar{X}}, \quad \mathcal{V}_{\bar{X}} \partial_{0} R^{(1)}(x \mid J)=\partial_{0} \hat{\phi}(x) \mathcal{V}_{\bar{X}}
$$

справедливым при $x^{0}=0$. Поскольку $\hat{\phi}(x)$ и $R^{(1)}(x \mid J)$ при $x \gtrsim \operatorname{supp} J$ удовлетворяют одному и тому же дифференциальному уравнению второго порядка, получаем свойство (4.8).

С точностью до числового множителя, зависящего от способа перенормировки, свойство (4.7) можно получить из пуанкаре-инвариантности полей. Действительно, операторы $U_{g}\left(u_{g} X \leftarrow X\right)$ определяются из (2.12) с точностью до мультипликативной константы, а оператор $\mathcal{V}_{u_{g}} U_{g} U_{\bar{X}}^{-1}$ удовлетворяет (2.12). Исследование числового множителя требует более детального анализа перенормировки преобразований Лоренца.

Отметим также, что формулы (4.8) и (4.9) позволяют получить полезные соотношения, выражаюшие квазиклассический оператор поля $\hat{\phi}(x \mid X)$ через 1-фформу $\Omega$. Обозначим для краткости

$$
\Omega\{\delta \Phi(\cdot)\}=\Omega(\delta \Phi, \delta \dot{\Phi})=\int d \mathbf{x}[\delta \dot{\Phi}(\mathbf{x}, 0) \hat{\phi}(\mathbf{x})-\delta \Phi(\mathbf{x}, 0) \hat{\pi}(\mathbf{x})],
$$

если $\delta \Phi$ - решение уравнения

$$
\partial_{\mu} \partial^{\mu} \delta \Phi+V^{\prime \prime}(\Phi(x)) \delta \Phi=0 .
$$


Как вытекает из (4.8), (4.9), (4.13),

$$
\int d x \hat{\phi}(x \mid X) \delta J(x)=\Omega\left\{\delta \Phi_{+}(\cdot)\right\}
$$

где $\delta \Phi_{+}$определяется из соотношений (4.10) и (4.12).

Соотношение (4.15) показывает, что аксиомы сушествования квазиклассического поля и его инвариантности не являются независимыми от остальных аксиом: если в качестве определения квазиклассического поля $\hat{\phi}(x \mid X)$ принять соотношение (4.15), его релятивистская инвариантность будет следовать из релятивистской инвариантности 1-формы $\Omega$.

\section{5. ЗАКЛЮЧЕНИЕ}

Как показано в работе, из первых принципов аксиоматического подхода при достаточно обших предположениях удается получить основные формулы классической и квазиклассической теории поля, включая принцип наименьшего действия, уравнения движения и коммутационные соотношения для квазиклассических полей.

Основной трудностью аксиоматического подхода к квантовой теории поля является построение нетривиальной модели, удовлетворяющей всем аксиомам. Поэтому при построении конкретных моделей квазиклассической теории поля имеет смысл выделять те свойства, которые сушественны в главном порядке по $h$, и проверять только их. Такими свойствами являются аксиомы А1-А6 в гамильтоновом подходе и дополнительно к ним свойства (2.23)-(2.26) - в ковариантном.

В настояшей работе рассмотрен только главньй порядок квазиклассического разложения. Обобщение приведенных конструкций, учитывающее поправки по $\sqrt{h}$, исследовано в нашей работе [23]. Там же изучена связь аксиом квазиклассической теории и $S$-матричного подхода в рамках теории возмущений, рассмотрены примеры исследования процессов распада и рассеяния частиц в данном подходе.

Автор надеется рассмотреть в последующих публикациях вопросы об аксиоматическом построении квазиклассических калибровочных теорий и ферми-полей и о взаимосвязи предложенных конструкций с моделями конструктивной теории поля.

Благодарности. Работа выполнена при финансовой поддержке РФФИ, проект № 02-01-01062.

\section{Список литературы}

[1] В. П. Маслов. Операторные методы. М.: Наука, 1973.

[2] В.П. Маслов. Комплексный метод ВКБ в нелинейных уравнениях. М.: Наука, 1977.

[3] В. П. Маслов. Теория возмущений и асимптотические методы. М.: МГУ, 1965.

[4] В.Г. Багров, В. В. Белов, И. М. Тернов. ТМФ. 1982. Т. 50. С. 390.

[5] В. П. Маслов, О. Ю. Шведов. Метод комплексного ростка в задаче многих частиц и квантовой теории поля. М.: УРСС, 2000.

[6] В.П. Маслов, О.Ю. Шведов. ТМФ. 1998. Т. 114. С. 233.

[7] O. Yu. Shvedov. J. Math. Phys. 2002. V. 43. P. 1809.

[8] Н. Н. Боголюбов, А. А. Логунов, А.И. Оксак, И. Т. Тодоров. Общие принципы квантовой теории поля. М.: Наука, 1987. 
[9] H. Lehmann, K. Symanzik, W. Zimmermann. Nuovo Cimento. 1957. V. 6. P. 319; K. Nishijima. Phys. Rev. 1960. V. 119. P. 485.

[10] Н. Н. Боголюбов, Д. В. Ширков. Введение в теорию квантованных полей. М.: Наука, 1984.

[11] Н. Н. Боголюбов, Б. В. Медведев, М. К. Поливанов. Вопросы теории дисперсионных соотношений. М.: Физматгиз, 1958; Б. В. Медведев, М.К. Поливанов, В.П. Павлов, А. Д. Суханов. ТМФ. 1972. Т. 13. С. 3.

[12] О. И. Завьялов. Перенормированные диаграммы Фейнмана. М.: Наука, 1979.

[13] А. А. Славнов, Л. Д. Фаддеев. Введение в квантовую теорию калибровочных полей. М.: Наука, 1988.

[14] Ю. Швингер. Частицы. Источники. Поля. М.: Мир, 1973.

[15] О. Ю. Шведов. Матем. заметки. 1999. Т. 65. С. 437; Матем. сб. 1999. Т. 190. № 10. С. 123.

[16] O. Yu. Shvedov. Ann. Phys. 2002. V. 296. P. 51.

[17] В. П. Маслов, О. Ю. Шведов. ТМФ. 1995. Т. 104. С. 479.

[18] A. Ashtekar, J. Lewandowski, D. Marolf, J. Mourao, T. Thiemann. J. Math. Phys. 1995. V. 36. P. 6456 ; D. Giulini, D. Marolf. Class. Q. Grav. 1999. V. 16. P. 2489; O. Yu. Shvedov. Ann. Phys. 2002. V. 302. P. 2.

[19] О. Ю. Шведов. ТМФ. 2003. Т. 136. С. 418; hep-th/0111265.

[20] R. Peierls. Proc. Roy. Soc. Lond. A. 1952. V. 214. P. 143.

[21] Б. С. ДеВитm. Динамическая теория групп и полей. М.: Наука, 1987.

[22] D. Marolf. Ann. Phys. 1994. V. 236. P. 392.

[23] O. Yu. Shvedov. An axiomatic approach to semiclassical field perturbation theory. hep-th/0412302.

Поступила в редакцию $16 . \mathrm{IX} .2004$ г., после доработки 18.III.2005 г. 